\title{
Brief report: nonsuicidal self-injury in adolescence: turning to the Internet for support
}

\section{ABSTRACT}

Research has highlighted the salience of online activity involving nonsuicidal self-injury (NSSI) among adolescents who self-injure. Most research in this area has been descriptive in nature, however, little is known about the precise activities by these youth and whether this varies by NSSI frequency. Thus, the present study examined the frequency of Internet site use for emotional health reasons and the specific online activities for emotional health reasons among adolescents who reported recent, past and no engagement in NSSI. One hundred and forty-two adolescents $\left(M_{\text {age }}=14.43, S D=.57 ; 64.1 \%\right.$ female $)$ completed a series of questionnaires to assess NSSI status and online activity. The findings revealed an interesting pattern of Internet use among adolescents who recently engaged in NSSI indicative of connecting (sharing and getting support) online, in contrast to those with a past history of selfinjury who reported online activity more similar to those with no history of self-injury. Research and clinical implications are discussed.

\section{KEYWORDS}

nonsuicidal self-injury; adolescence; internet; online activity; emotional health

Nonsuicidal self-injury (NSSI), the deliberate damaging of one's own body tissue (e.g. self-cutting, burning, bruising) without suicidal intent, is a critical adolescent concern. Indeed, research indicates that NSSI associates with myriad mental health difficulties and elevates suicide risk (Lewis \& Heath, 2015). Recently, there is a growing body of literature that has investigated the potential impact of online activity around NSSI for those who engage in selfharm behaviors (Lewis \& Seko, 2016); however, little is known about the specific nature of online activities and NSSI among those youth involved. 
Preliminary studies of examining youth NSSI and Internet use suggest that adolescents who engage in NSSI may use the Internet more often and for different reasons than their peers who do not self-injure (Heath, Toste, \& McLouth, 2010; Mitchell \& Ybarra, 2007). Generally, adolescent Internet users go online to obtain health information and to seek help for emotional difficulties, suggesting the Internet represents a resource for socially sensitive topics (e.g. Naslund, Grande, Aschbrenner, \& Elwyn, 2014). This may be especially relevant for young people who feel stigmatized or isolated. Indeed, growing research indicates that youth and emerging adults who self-injure may view the Internet as providing a low-risk venue for finding information and receiving social support and validation (Lewis \& Michal, 2014; Lewis, Rosenrot, \& Messner, 2012; Lewis \& Seko, 2016). Adding support to this is research indicating that accessing NSSI information and seeking emotional support may motivate NSSI ecommunication among young adults who self-injure; this may be particularly relevant for female users (Lewis \& Michal, 2014). To date, however, few efforts directly examined this in adolescence, when NSSI and online activity may be especially relevant.

One study examining differences between online and offline help-seeking among youth who self-injure found that youth who were less likely to seek help professionally, were more likely to seek help for NSSI online (Frost \& Casey, 2016). Notably, these individuals also reported higher psychological distress and were more likely to have required medical help versus those who did not seek online NSSI help. Despite these findings, adolescents who engage in NSSI often do not come to the attention of healthcare professionals (Hawton, Rodham, Evans, \& Harriss, 2009) and may disapprove of professionals who offer support (Rodham, Gavin, Lewis, St. Denis, \& Bandalli, 2013).

The recent incorporation of NSSI in Section 3 of the DSM-5 as a disorder warranting 
further research has called for a greater understanding of the behaviour. Under the proposed criteria, individuals must have self-injured within the last year (American Psychiatric Association, 2013). Current evidence suggests that those reporting recent NSSI (i.e. past year) differ from those with a more distal NSSI history. However, very few studies have explored the differences in individuals with past year NSSI as compared to those with a history of NSSI prior to one year (e.g. Muehlenkamp \& Brausch, 2016; Taliaferro \& Muehlenkamp, 2015). In one study, Taliaferro and Muehlenkamp (2015) found that in a community sample of high school students, gender, younger age, greater depressive symptoms, greater anxiety symptoms and more hopelessness distinguished adolescents with recent NSSI from those with a history of NSSI. Similarly, Muehlenkamp and Brausch (2016) found that engaging in NSSI within the past year, compared to more than one year ago, was associated with greater depression and suicidal ideation among undergraduate students.

Despite the evidence that those with recent self-injury differ substantially from those with a history of self-injury, it remains unclear whether these groups might differ based on Internet use. This is critical as they may have different needs and different motivations for online activity. For example, preliminary research suggests that differences exist in reasons for starting, temporarily stopping and continuing NSSI e-communication (Lewis \& Michal, 2014). In addition, there is research suggesting that unlike the motivations for engaging in NSSI that most commonly include affect regulation, relief from distress and communication of distress (e.g. Klonsky, Muehlenkamp, Lewis, \& Walsh, 2011), the motivations for posting about NSSI may be more outwardly directed (Rodham et al., 2013). Consistently, individuals posting NSSI material online may be targeting different audiences and for different reasons (e.g. Lewis \& Baker, 2011), which might reflect the recency of self-injury. 
Taken together, accessing health-related information and support are of particular importance when considering the needs of individuals engaging in online NSSI activities (Lewis $\&$ Seko, 2016). Thus, the first aim of the present study was to examine the frequency of Internet use for emotional health reasons among adolescents who report recent (past year) NSSI, more distal NSSI and adolescents with no NSSI history. The comparison group of no NSSI history was a group of at-risk adolescents who reported other risky behaviours to cope (e.g. drug use, alcohol use, promiscuous or unprotected sexual activities). A comparison of at-risk youth was chosen as these youth may be equally expected to seek online support around their risky behaviours. It was hypothesized that adolescents reporting recent NSSI would report more Internet use for emotional health reasons versus those reporting more distal or no NSSI and that those reporting more distal NSSI would report more Internet use for emotional health reasons than those who have not self-injured. It was also expected that females across all groups would report more frequent Internet use compared to males. The study's second aim was to examine the nature of Internet use for emotional health reasons among adolescents who report recent NSSI, distal, and no history of NSSI; specifically, what are (a) the reported motivations for the online activity for emotional health reasons, (b) common emotional health topics of interest and (c) the online activities used for emotional health reasons across groups. Given the exploratory nature of this objective, no hypotheses were made.

\section{Method}

\section{Participants}

Participants were drawn from a larger pool of participants in Grade $9(N=764)$ from 17 high schools in an urban area taking part in a project investigating stress and coping strategies. Students in earlier grades were not included due to concerns raised by schools and the ethics 
research board around probing for NSSI online activity in that it may be suggestive for younger students.

All participants for the current study were selected for follow-up interviews based on screening items for engagement in NSSI, and/or risky behaviours (e.g. drug use, alcohol use, gambling, promiscuity) to cope with stress. Only adolescents who reported engaging in the behaviour(s) that correspond to the definition of NSSI (International Society for the Study of Self-Injury, 2007) were classified in the NSSI groups. One hundred and forty-two students (64.1\% female), with a mean age of $14.43(S D=.57)$ were selected for interviews based on initial NSSI screening (described below).

\section{Measures}

How I Deal with Stress Questionnaire (HIDS; Heath \& Ross, 2007). The HIDS was used to collect demographic information and screen for NSSI. Participants rate their use of 31 healthy and unhealthy stress coping strategies on a four-point Likert scale; those who report having intentionally hurting themselves physically are then prompted to complete a follow-up section assessing NSSI. Toste, Christie, and Heath (2011) have found high test-retest reliability for the NSSI screening item $(r=.83)$ after 6 months.

Self-Injurious Thoughts and Behaviors Interview (SITBI; Nock, Holmberg, Photos, \& Michel, 2007). The SITBI is a semi-structured interview assessing NSSI thoughts and behaviours and was used to confirm NSSI status. Nock et al. (2007) found that the NSSI module had strong test-retest reliability (average $\kappa=.70$, intra-class correlation coefficient .44 ) after 6 months. Emotional Health Online Behaviour Assessment (EHOBA). Informed by past research (e.g. Rodham et al., 2013; Whitlock, Powers, \& Eckenrode, 2006), an 8-item questionnaire was created by the researchers given the absence of a measure assessing online health behaviour. It 
comprises two sections, tapping: (a) frequency of Internet use (i.e. how often participants used nine websites [e.g. Facebook, Instagram, Tumblr, YouTube, Wikipedia] rated on a five-point Likert from never to very often) for emotional health reasons, and (b) nature of online activities regarding emotional health (i.e. when you go online for emotional health reasons, what topics are you interested in [e.g. stress, coping, self-injury, anxiety, depression, anger, eating disorders, sadness, other]?) and motivations for engaging in these activities (i.e. Why do you go online for emotional health reasons [e.g. to share my experience/story with others; to get help from a mental health profession, other]?). For the latter section, participants are instructed to check off the items that are applicable to them. The websites included were chosen based on popularity, in terms of traffic they accrued at the time of the study (searched http://www.alexa.com/ in 2015), relevance to youth (Lenhart, 2015) and relevance to NSSI (Duggan, Heath, Lewis, \& Baxter, 2012; Lewis, Heath, St Denis, \& Noble, 2011; Lewis \& Seko, 2016). Internal consistency values for each section $(\alpha=.70, .74$, respectively) were acceptable.

\section{Procedure}

After receiving ethical approval from McGill University and following parental consent and student assent, students completed screening questionnaires in 45-min sessions during class time. Adolescents who endorsed items "I do risky things to cope with stress" and/or "I physically hurt myself on purpose" on the HIDS were selected for individual follow-up to further confirm NSSI status. The follow-up assessments consisted of another battery of questionnaires, including the SITBI, EHOBA and ending with a suicide risk assessment. The interviews were conducted in private rooms by doctoral students in school psychology trained by a psychologist on suicide risk assessments. Confidentiality was broken only in the event that the participant indicated to the interviewer that they were suicidal or at risk for harming someone else, in which case, the 
participant was informed and transitioned to a pre-deter- mined school mental health professional (i.e. school psychologist, school counsellor) that was aware of the nature of the project. Adolescents reporting NSSI in the past 12 months on the HIDS and the SITBI/follow-up interviews were placed in the recent NSSI group $(n=58 ; 79 \%$ female) while those reporting NSSI prior to this were placed in the past NSSI group $(n=28 ; 50 \%$ female). A comparison group of at-risk youth reporting risky behaviours, yet no NSSI $(n=56 ; 55 \%$ female $)$ was also formed to ensure that students interviewed were not identifiable to peers as targeted for difficulties. All students who completed an individual follow-up interview received a ten-dollar gift card. Furthermore, all participating students' names were entered in a draw for four $\$ 50$ gift cards.

\section{Results}

\section{Frequency of Internet use for emotional health reasons across NSSI groups}

A principal component analysis (PCA) was computed to group individual Internet sites used by participants. Following this, a one-way multivariate analysis of variance (MANOVA) and subsequent univariate ANOVAs were performed, comparing recent NSSI, past NSSI and no NSSI groups on these PCA factor scores. The PCA revealed three components with eigenvalues greater than one. Visual inspection of the scree plot indicated that two components should be retained (Meyers, Gamst, \& Guarino, 2006). The two-component solution explained $41.82 \%$ of the total variance. Given the correlation among factors, a Direct-Oblimin orthogonal rotation guided interpretation (Tabachnick \& Fidell, 2007). To this end, the data were consistent with the expected factors of the EHOBA, with Component 1 being social media sites (e.g. Instagram, Twitter, Facebook) and Component 2, non-social media sites (e.g. Wikipedia, Tumblr, Pinterest). The 3 (NSSI groups) by 2 (gender) MANOVA for frequency of social media and non-social 
media site usage revealed a significant overall multivariate NSSI group difference, $F=(4,142)=$ 2.60, $p=.039 ;$ Wilks' $\Lambda=.92 ;$ partial $\eta^{2}=.037$, with no gender or interaction effect (Table 1). Follow-up univariate ANOVAs revealed a significant difference between NSSI groups for frequency of social media site usage $F=(2,142)=5.02, p=.008$, partial $\eta^{2}=.07$; no difference was found for non-social media sites (Table 2). Follow-up Tukey post hoc tests revealed that the recent NSSI group $(M=1.00, S D=.79)$ had significantly higher mean scores on use of social media sites than the past NSSI group $(M=.56, S D=.61), p=.014$, and significantly higher mean scores than the no NSSI comparison group $(M=.48, S D=.57), p=.000$. However, no difference was found between the past and no NSSI group (Table 3).

Online activity for emotional health reasons across NSSI groups

Results of Chi-square tests examining online activities between NSSI groups revealed a statistically significant association between NSSI groups and going online to support others, $\chi^{2}$ $(2)=22.52, p=.000, p h i=.41$, and "to get support from other people who are dealing with the same thing as me", $\chi^{2}(2)=12.17, p=.002, p h i=.30$. Specifically, the recent NSSI group was more likely to get/give support to others dealing with the same difficulties as them, compared to both the past and no NSSI groups.

A significant association was also found between NSSI groups and reading information, $\chi^{2}(2)=6.03, p=.049, p h i=.21$, looking at pictures, $\chi^{2}(2)=7.20, p=.027, p h i=.22$, looking at videos, $\chi^{2}(2)=6.180, p=.046, p h i=.21$ and blogging, $\chi^{2}(2)=12.72, p=.002, p h i=.30$, indicating that the recent NSSI group was more likely to engage in all of these activities compared to the past and no NSSI groups. In addition, a significant association was found between NSSI group and interest in depression, $\chi^{2}(2)=16.77, p=.000, p h i=.34$, self-injury, $\chi^{2}$ $(2)=26.57, p=.000, p h i=.43$ and eating disorders, $\chi^{2}(2)=9.752, p=.008 p h i=.262$, 
indicating that the recent NSSI group was more likely to report interest in these topics compared to the past and no NSSI groups. Finally, the distal NSSI group reported more interest in the topic of sadness versus the other groups, $\chi^{2}(2)=9.93, p=.007, p h i=.26$.

\section{Discussion}

This is the first study to document that Internet use for emotional health reasons by adolescents who self-injure differs on the basis of NSSI recency. These findings build on previous research indicating that obtainment and provision of support online may, in part, drive online NSSI activity (see Lewis \& Seko, 2016). In particular, social media websites may have particular relevance when NSSI has been more recently enacted. The present study also adds to research indicating that those who report a more distal NSSI history are more similar to those with no history than those with a recent NSSI history (Taliaferro \& Muehlenkamp, 2015) by suggesting that this may extend to online activities. Despite some research indicating that more females use the Internet to obtain emotional support for NSSI (Lewis \& Michal, 2014), the present study found no such gender differences. Further work is thus needed to better understand the extent to which these differences exist and why.

The apparent centrality of peer-to-peer online support among youth who recently self-injure is notable. This finding not only aligns with a growing body of research pointing to the - at times - positive role of the Internet among those who self-injure (Lewis \& Seko, 2016) but also to a preference for peer versus professional support for NSSI (Rodham et al., 2013). Indeed, participants in the current study seldom reported using the Internet to obtain professional support or resources. That youth rarely seek professional support online requires further investigation; for instance, it would be helpful to know why the efforts of many professionally driven NSSI websites (e.g. SAFE Alternatives) may not be utilized by youth. Offline, only a small proportion 
of youth who self-injure seek help from mental health pro- fessionals and prefer to disclose their NSSI to a close friend or peer (Berger, Hasking, \& Martin, 2013; Muehlenkamp, Walsh, \& McDade, 2010). Thus, the tendency to confide in peers rather than professionals may be an extension of offline-help-seeking among youth who self-injure. Alternatively, there may be other factors that deter youth from seeking professional help online (e.g. not being aware of existing web resources, unappealing content). Future studies should aim to examine both online and offline professional-help-seeking among the same sample of youth.

Finally, youth who report recent NSSI express greater interest in depression and eating disorders versus youth with past or no NSSI histories, which may indicate an overall greater level of mental health difficulties. If so, it would to be consistent with research indicating that youth with current NSSI are characterized by elevated psychopathology (e.g. Muehlenkamp \& Brausch, 2016; Taliaferro \& Muehlenkamp, 2015) and that youth seeking online NSSI support have greater levels of distress overall (Frost \& Casey, 2016). Evidently, relative to youth with past or no NSSI history, those who report current NSSI reported greater interest in seeking online NSSI material. However, the nature of what they are seeking remains unclear and warrants further investigation. Interestingly, those reporting past NSSI reported similar, and at times greater interest in obtaining information about negative emotions, than the recent NSSI group. Current literature suggests that NSSI often co-occurs with a number of psychological difficulties (e.g. emotion dysregulation, self-hatred, depression, problematic body image; Fox et al., 2015; Hankin \& Abela, 2011; Muehlenkamp \& Brausch, 2012). Thus, a possible interpretation of this pattern is that while those with a history of NSSI are no longer as interested in self-injury material they may be struggling with on-going mental health challenges. 


\section{Limitations}

Although NSSI engagement was rigorously confirmed, caution should be taken when drawing conclusions about distal NSSI indicating full recovery; indeed, NSSI may be fleeting and only occur in the context of acute stress (e.g. Berger et al., 2013). Future research should ask directly about recovery and employ longitudinal assessment of NSSI to delineate those who temporarily versus permanently cease NSSI. Additional qualitative information (e.g. open- ended questions, interviews) regarding online support seeking would also be beneficial in further understanding reasons youth partake in online NSSI activities. Of note, not all students who indicated engaging in NSSI on the screening questionnaire disclosed NSSI during the follow-up interviews. While this was not directly examined in the current study, similar findings have been noted in previous literature (e.g. Bjärehed, Pettersson, Wångby-Lundh, \& Lundh, 2013) and raise important considerations around youths' comfort level around discussing NSSI.

According to the present findings, adolescents who engage in NSSI report a different pattern of online activity for emotional health relative to adolescents who are at-risk for other forms of unhealthy coping. While using an at-risk adolescent comparison group is reasonable as they are likely to also be seeking online support, it would be helpful in future to have a third more typical comparison group as well. Moreover, the term emotional health was used as a broader term for any emotional concerns that adolescents may have, yet this may have had less relevance and missed part of the target population. Specifically, research has shown that a number of adolescents have difficulty describing "why” they self-injure (Klonsky et al., 2011). Thus, for those who do not understand why they are engaging in the behaviour, and specifically what purposes it serves (e.g. emotional relief ), the questions asked may have been misleading. Therefore, replication with more specific questions regarding self-injury, rather than a global 
emotional health concept may be more generalizable.

\section{Conclusion and clinical implications}

The present findings may have several implications for professionals who work with youth who engage in NSSI. First, the present findings highlight the importance of professionals being aware of the range of online activities that youth who are struggling with NSSI may be engaging in and the importance of these for their support. The use of published guidelines for assessing, intervening, and monitoring adolescents' online NSSI activity may be useful in these contexts (Lewis, Heath, Michal, \& Duggan, 2012). Second, in light of the current results suggesting that those who recently self-injure report seeking support online, professionals should be prepared to assess and understand the extent and quality of online support youth are receiving as well as working with youth to develop offline supports. Finally, given that youth may prefer

online support for NSSI, it is also critical to ensure that they have access to good quality websites that are recovery-oriented and that encourage offline forms of help-seeking. 
This is an Accepted Manuscript of an article published by Taylor \& Francis in 'Counselling Psychology Quarterly' on 2018-01-21, available online: https://www.tandfonline.com/10.1080/09515070.2018.1427556.

\section{References}

American Psychiatric Association. (2013). Diagnostic and Statistical Manual of Mental Disorders. (5th ed.). Arlington, VA: American Psychiatric Association.

Berger, E., Hasking, P., \& Martin, G. (2013). “Listen to them”: Adolescents’ views on helping young people who self-injure. Journal of Adolescence, 36(5), 935-945.

Bjärehed, J., Pettersson, K., Wångby-Lundh, M., \& Lundh, L. G. (2013). Examining the acceptability, attractiveness, and effects of a school-based validating interview for adolescents who selfinjure. The Journal of School Nursing, 29(3), 225-234.

Duggan, J. M., Heath, N.L., Lewis, S. P., \& Baxter, A. L. (2012). An examination of the scope and nature of non-suicidal self-injury online activities: Implications for school mental health professionals. School Mental Health, 4(1), 56-67.

Fox, K. R.,Franklin, J. C., Ribeiro, J.D., Kleiman, E. M., Bentley, K. H., \& Nock, M. K. (2015). Metaanalysis of risk factors for nonsuicidal self-injury. Clinical Psychology Review, 42, 156167.

Frost, M., \& Casey,L. (2016). Who seeks help online for self-injury? Archives of Suicide Research, 20(1), 69-79.

Hankin, B. L. \& Abela, J. R. (2011). Nonsuicidal self-injury in adolescence: Prospective rates and risk factors in a 2 1/2 year longitudinal study. Psychiatry Research, 186(1), 65-70.

Hawton, K., Rodham, K., Evans, E., \& Harriss, L. (2009). Adolescents who self harm: A comparison of those who go to hospital and those who do not. Child and Adolescent Mental Health, 14(1), 2430.

Heath, N. L., \& Ross, S. (2007). How I deal with stress. Unpublished instrument. Montreal, QC :McGill 
University.

Heath, N. L., Toste, J. R. \& McLouth, R. (2010). Unpublished raw data.

International Society for the Study of Self-Injury. (2007). Definition ofnon-suicidal self-injury.

Retrieved from http://www.itriples.org/isss-aboutself-i.html

Klonsky, E. D., Muehlenkamp, J. J., Lewis, S. P., \& Walsh, B. (2011). Nonsuicidal self-injury. Cambridge, MA: Hogrefe \& Huber. Lenhart, A. (2015, April 8). Teens, socialmedia \& technologyoverview 2015. Retrieved June 24, 2016, from http://www.pewinternet.org/2015/04/09/teens-socialmedia-technology-2015/

Lewis, S. P., \& Baker, T. G. (2011). The possible risks of self-injury web sites: Acontent analysis. Archives of Suicide Research, 15(4), 390-396.

Lewis, S.P.,\& Heath, N.L.(2015). Nonsuicidal self-Injury among youth. The Journal of Pediatrics, 166(3), 526-530.

Lewis, S. P., Heath, N. L., Michal, N. J., \& Duggan, J. M. (2012). Non-suicidal self-injury, youth, and the Internet: What mental health professionals need toknow. ChildandAdolescent Psychiatryand Mental Health, 6(1), 13.

Lewis, S. P., Heath, N. L., St Denis, J. M., \& Noble, R. (2011). The scope of nonsuicidal self-injury on YouTube. Pediatrics, 127(3), e552-e557.

Lewis, S. P., \& Michal, N. J. (2014). Start, stop, and continue: Preliminary insight into the appeal of selfinjury e-communities. Journal of Health Psychology, 21(2), 250-260.

Lewis, S. P., Rosenrot, S. A., \& Messner, M. A. (2012). Seeking validation in unlikely places: The nature of online questions about non-suicidal self-injury.Archives of Suicide Research, 16(3), 263272.

Lewis, S.P.,\& Seko, Y.(2016). A double-edged sword: A review of benefits and risks of online nonsuicidal 
self-injury activities. Journal of Clinical Psychology, 72(3), 249-262.

Meyers, L. S., Gamst, G., \& Guarino, A. J. (2006). Applied multivariate research: Design and Interpretation. Thousand Oaks, CA: Sage.

Mitchell, K. J., \& Ybarra, M. L. (2007). Online behavior of youth who engage in self-harm provides clues for preventive intervention. Preventive Medicine, 45(5), 392-396.

Muehlenkamp, J. J. \& Brausch, A. M.(2012). Body image as a mediator of non-suicidal self-injury in adolescents. Journal of Adolescence, 35(1), 1-9.

Muehlenkamp, J. J., \& Brausch, A. M. (2016). Reconsidering criterion a for the diagnosis of non-suicidal self-injury disorder. Journal of Psychopathology and Behavioral Assessment, 38(4), 547-558.

Muehlenkamp, J. J., Walsh, B. W., \& McDade, M. (2010). Preventing non-suicidal self-injury in adolescents: The signs of self-injury program. Journal of Youth and Adolescence, 39(3), 306-314.

Naslund, J. A., Grande, S. W., Aschbrenner, K. A., \& Elwyn, G. (2014). Naturally occurring peer support through social media: The experiences of individuals with severe mental illness using YouTube. PLOS one, 9(10), e110171.

Nock, M. K., Holmberg, E. B., Photos, V.I., \& Michel, B. D. (2007). Self-injurious thoughts and behaviors interview: Development, reliability, and validity in an adolescent sample. PsychologicalAssessment, 19(3), 309.

Rodham, K., Gavin, J., Lewis, S. P., St. Denis, J. M., \& Bandalli, P.(2013). An investigation of the motivations driving the online representation of self-injury: A thematic analysis. Archives of Suicide Research, 17(2), 173-183.

Tabachnick, B. G., \& Fidell, L. S. (2007). Multivariate analysis of variance and covariance. Using Multivariate Statistics, 3, 402-407.

Taliaferro, L. A., \& Muehlenkamp, J. J. (2015). Factors associated with current versus lifetime self-injury 
among high school and collegestudents. Suicide and Life-Threatening Behavior, 45(1), 8497.

Toste, J.R., Christie, M., \& Heath, N.L. (2011). How doIdeal with stress (HIDS): Evidenceforthe use of an NSSI screening questionnaire among young adults. New York, NY: International Society for the Study of Self-Injury.

Whitlock, J.L.,Powers, J.L., \& Eckenrode, J. (2006). The virtual cutting edge: The Internet andadolescent self-injury. Developmental Psychology, 42(3), 407. 


\section{Tables and Figures}

Table1. Main effects of internetuse for emotional health reasons across groups and gender.

\begin{tabular}{lcccc}
\hline Main effect & df & Wilks & $\eta^{2}$ & $p$ \\
& & & & \\
\hline nssi group & & & \\
& 4 & .92 & .04 & .03 \\
gender & 2 & .96 & .04 & .057 \\
nssi group $\times$ gender & 4 & .99 & .01 & .873 \\
\hline
\end{tabular}

note: adolescents reporting nssi in the past 12 months were placed in the recent nssi group; adolescents reporting nssi prior to 12 months were placed in the Past nssi group; adolescents reporting no nssi were placed in the nonssi group.

$* p<.05$.

Table 2. univariate effects of internet use for emotional health reasons and gender on nssi groups.

\begin{tabular}{lccccc}
\hline $\begin{array}{l}\text { Dependent } \\
\text { variables }\end{array}$ & df & $F$ & $\eta^{2}$ & Observed & $p$ \\
\hline social media sites & 2 & 5.02 & .0 & .81 & .00 \\
& & & 7 & & $8^{*}$ \\
non social media & 2 & .75 & .0 & .18 & .473 \\
sites & & & 1 & & \\
\hline
\end{tabular}


$* p<.05$.

Table 3. nssi group means and standard deviations for internet use for emotional health.

\begin{tabular}{lcccccc} 
& \multicolumn{2}{c}{ Recent NSSI $(n=58)$} & \multicolumn{2}{l}{ PastNSSI $(n=28)$} & \multicolumn{2}{c}{ No NSSI $(n=56)$} \\
\cline { 2 - 7 } Variables & $M$ & $S D$ & $M$ & $S D$ & $M$ & $S D$ \\
\hline social media & 1.0 & .79 & .5 & .61 & .48 & .5 \\
& 0 & & 6 & & & 7 \\
non social & .26 & .43 & .1 & .33 & .16 & .2 \\
media & & & 9 & & & 8
\end{tabular}

note: adolescents reporting nssi in the past 12 months were placed in the recent nssi group; adolescents reporting nssi prior to 12 months were placed in the Past nssi group; adolescents reporting no nssi were placed in the no nssi group. 